\title{
ECODRIVE TRAINING DELIVERS SUBSTANTIAL FUEL SAVINGS FOR HEAVY VEHICLE DRIVERS
}

\author{
Mark A. Symmons ${ }^{1}$ and Geoff Rose ${ }^{2}$ \\ ${ }^{1}$ Psychological Studies \\ ${ }^{2}$ Institute of Transport Studies \\ Monash University \\ Churchill, Australia \\ Email: mark.symmons@muarc.monash.edu.au
}

\begin{abstract}
Summary: A small group of heavy vehicle drivers underwent an ecodrive training course. Their driving was assessed for various ecodrive variables as they completed an 18 mile circuit in normal traffic immediately after the course and again 6 and 12 weeks later. Compared to pre-course measures, these drivers reduced their fuel consumption by an average of $27 \%$, the number of gear changes by $29 \%$, and the number of brake applications by $41 \%$, though not all differences were statistically significant due to the size of this pilot and large driver variability. Importantly, the improvements were not offset by increases in the time taken to complete the circuit. At the 6 week point a control group was also assessed, and they used more fuel and more gear changes, and applied their brakes more often than the control group. Safety variables were inconclusive. A larger, in-service trial is warranted.
\end{abstract}

\section{BACKGROUND}

The concept of ecodriving has been in existence for at least 15 years, and its use has been widespread in Europe. In the UK more than 12,000 heavy vehicle drivers have received training from one scheme alone, with fuel savings of the order of $10 \%$ (SAFED, 2007). The primary thrust of ecodriving is a smoother driving style - 'gliding' or 'flowing' through traffic - with the principal aim of reducing fuel consumption and emissions. These savings are realised with behaviours that include shifting up through the gears as soon as possible and skipping gears when appropriate, maintaining a steady speed in the highest gear possible, and maintaining appropriate tyre pressures and servicing schedules. It is also critical to avoid heavy and/or sudden acceleration or braking and to look as far ahead down the traffic stream as possible in order to anticipate the actions of other drivers and predict likely changes and interruptions to the traffic flow. This more defensive or anticipatory driving style should also serve to reduce the likelihood of being involved in a crash.

Much of the previous research and implementation in this area has focused on passenger cars rather than heavy vehicles. While there are fewer heavy vehicles, their average annual distance of travel is significantly larger than that for cars - in 2007 the average Australian passenger vehicle travelled 13,700 kilometres (8,220 miles), while rigid trucks and articulated trucks travelled 22,000 (13,200 miles) and 93,200 kilometres (55,920 miles) respectively (ABS, 2008). Additionally, heavy vehicles use substantially more fuel per kilometre travelled -28.5 and 54.6 litres per 100 kilometres $(8.3-4.3 \mathrm{mpg})$ for rigid and articulated heavy vehicles respectively, versus an average of 11.5 litres per 100 kilometres $(20.5 \mathrm{mpg})$ for passenger vehicles. 
Accordingly, the potential benefits in terms of fuel saved from more efficient driving could be greater on a per-driver or per-vehicle basis for heavy vehicle use.

A wide range of ecodrive outcomes for heavy vehicle training programs is matched by the variety of methods of training and assessment used. For example, Parks and Reed (2005) showed drivers an instructional ecodrive video and tested their performance with a heavy vehicle simulator; Zarkadoula, Zoidis and Tritopoulou (2007) instrumented a pair of buses to collect inservice data from three drivers as they negotiated normal traffic after completing training; and Holcim (2005) administered a full-day course and tested drivers on a set in-traffic circuit. Most trials make before versus after comparisons, which can be confounded by seasonality effects (Coyle \& Brown, 2004). According to af Wahlberg (2007), much of the previous ecodrive research is riddled with flaws and unsubstantiated claims. Employing a control group is a critical component of any rigorous trial - a consideration not taken into account by most published trials

With no evidence that a rigorous trial of ecodrive-type training had previously been conducted in Australia, the current project was designed as a case-study to test the potential of ecodrive training here and garner support for a larger program of training and evaluation. To maximise rigour and address af Wahlberg's (2007) criticisms, a control group was included and testing carried out in "normal" traffic conditions.

\section{METHOD}

Two B-double trucks were used in the field trial (see Figure 1). They were 25 metres (82 feet) long, 550 horsepower and fully loaded weighed 68 tonne (75 ton).

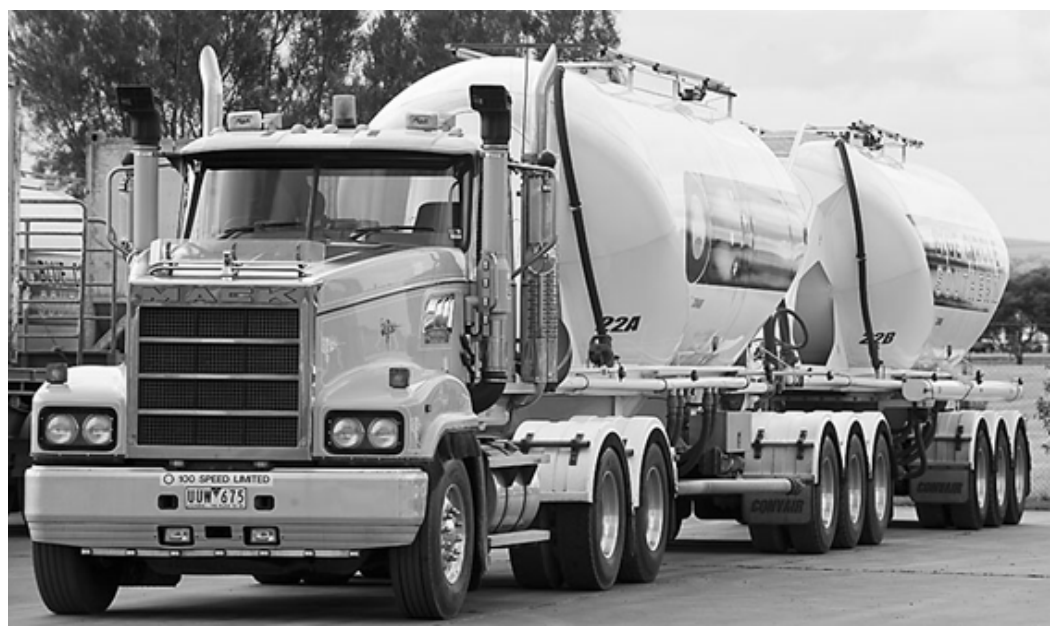

Figure 1. Mack B-double cement tankers used in the trial

A $30 \mathrm{~km}$ (18.6 mile) outer suburban circuit was used as an on-road test track, starting and finishing at a fuelling station. The route included a $100 \mathrm{~km} / \mathrm{h}(60 \mathrm{mph})$ freeway section as well as $80 \mathrm{~km} / \mathrm{h}(50 \mathrm{mph})$ urban and semi-rural arterial roads, and a $50 \mathrm{~km} / \mathrm{h}(31 \mathrm{mph}) \mathrm{section}$ through a strip shopping zone and residential areas. The drivers were all sufficiently familiar with the route they did not need to be directed. 
The training primarily consisted of a classroom-based theory session that lasted for approximately one hour. The course facilitator particularly focused on progressive gear shifting and progressive braking, "flowing" the vehicle and forward scanning of the road ahead. Drivers also discussed torque and power curves for modern diesel engines so that they might understand the theory underpinning the ecodrive concepts.

Twelve drivers were randomly selected from a pool of thirty across three company depots. They were randomly assigned to three groups: a full-course training group, a classroom-only training group, and a no-training control group. The full-course training drivers each drove a circuit of the test route before the classroom theory session. The classroom-only group did not complete a pre-drive before the classroom theory session, and the control group did not complete a pre-drive nor did they participate in any training.

As well as total fuel consumed travelling the circuit and circuit completion time, a number of other variables were collected by an observer from the passenger seat, including number of gear changes, instances of over revving $(1800+\mathrm{rpm})$, brake applications, and instances of insufficient scanning ahead and insufficient following distance. The drivers knew what data was being recorded by the in-cab observers, but did not receive feedback while driving. Data was collected for the full-course group before and immediately after their classroom session, and again at six and twelve weeks after training. Due to logistical and funding constraints the control and classroom-only groups were assessed at the six-week point only.

\section{RESULTS}

The results for the full-course group, immediately prior to and after the classroom session and at six and 12 weeks post-course are presented in Table 1.

Table 1. Means \& standard deviations for fuel consumption, brake applications, gear changes \& circuit completion time for the fully trained group at each test interval

\begin{tabular}{lcccccccc}
\hline Time & \multicolumn{2}{c}{ Fuel consumption } & \multicolumn{2}{c}{ Brake applications } & \multicolumn{2}{c}{ Gear changes } & \multicolumn{2}{c}{ Circuit time (mins) } \\
& Mean & SD & Mean & SD & Mean & SD & Mean & SD \\
\hline Pre-course & 26.4 & 5.8 & 32.3 & 8.8 & 74.0 & 18.1 & 35.1 & 0.2 \\
Post-course & 19.2 & 4.8 & 20.8 & 4.8 & 51.8 & 13.9 & 35.2 & 1.1 \\
6 wks post & 20.1 & 3.4 & 18.8 & 1.7 & 55.0 & 5.7 & 33.8 & 0.7 \\
12 wks post & 19.3 & 4.5 & 17.5 & 6.8 & 50.8 & 7.1 & 35.3 & 3.0 \\
\hline
\end{tabular}

On average, fuel consumption for the drivers who completed the full course decreased by $27 \%$ between the pre-course drive and the post-test drives, with the biggest drop immediately after the training. Importantly, the decrease was maintained at the 6- and 12-week points. However, despite the magnitude of this reduction, a within-groups ANOVA statistical test indicated that the this difference was not statistically significant $[F(3,9)=2 ; p>0.05]$, though the difference between the pre- $(M=26.4$ litres) and immediately post-course $(M=19.2$ litres $)$ approached significance according to post-hoc testing $(p=0.05)$.

The fully trained drivers reduced their instances of braking by an average of $41 \%$, ranging from a $36 \%$ reduction immediately post-course to a $46 \%$ reduction 12 weeks after training, suggesting a progressive improvement. This reduction was statistically significant $[F(3,9)=4.1 ; p<0.05]$. In 
post-hoc testing the differences between pre-course and immediately post-training ( $M=32.3$ vs. $M=20.8$ braking episodes respectively) and between pre-course and six weeks post-training ( $M=32.3$ vs. $M=18.8$ braking episodes respectively) both approached statistical significance ( $p=0.05$ in both instances).

Drivers in the full course also reduced the number of gear changes they employed to complete the circuit substantially, by an average of $29 \%$. This reduction was maintained through the 12 weeks of follow-up testing. The decrease was found to be statistically significant $[F(3,9)=7.9$; $p<0.01]$, and post-hoc testing revealed a significant difference between pre-course and immediately post-course gear changes ( $M=74$ vs. $M=51.8$ changes respectively, $p<0.01)$ and precourse and 12 weeks post-course gear changes ( $M=74$ vs. $M=50.8$ changes respectively, $p<0.05)$.

There was very little variation (and no statistical difference) in the average time taken to complete the circuits, with a maximum difference of one-and-a-third minutes $(3.7 \%)$ from the pre-course times. An overall average circuit time of 35 minutes corresponds to an average speed around the $30 \mathrm{~km}$ circuit of $51 \mathrm{~km} / \mathrm{h}(31 \mathrm{mph})$.

Table 2 contains the data for the full-course group, the classroom-only group and the control group at six weeks post-course.

Table 2. Means \& deviations for fuel consumption, brake applications \& gear changes for each of the experimental groups at 6 weeks post-course

\begin{tabular}{lcccccrrr}
\hline Group & \multicolumn{2}{c}{ Fuel consumption (L) } & \multicolumn{2}{c}{ Brake applications } & \multicolumn{2}{c}{ Gear changes } & \multicolumn{2}{c}{ Circuit time (mins) } \\
& Mean & Std Dev & Mean & Std Dev & Mean & Std Dev & Mean & Std Dev \\
\hline Trained & 20.1 & 3.4 & 18.8 & 1.7 & 55.0 & 5.7 & 33.8 & 0.7 \\
Classroom & 26.8 & 3.3 & 21.5 & 5.6 & 83.3 & 15.6 & 36.0 & 2.2 \\
Control & 27.5 & 2.6 & 27.0 & 5.2 & 68.3 & 6.8 & 35.2 & 1.8 \\
\hline
\end{tabular}

At the six-week point the difference in fuel consumption was significant [between-groups ANOVA $F(2,9)=7 ; p<0.05]$ such that the fully trained drivers differed from both the classroom and control groups $(M=20.1$ litres vs. $M=26.8$ litres, $p<0.05$ and $M=20.1$ litres vs. $M=27.5$ litres, $p<0.01$ respectively). The classroom group did not differ significantly from the control group.

A comparison of gear changes across the groups at six weeks post-training also revealed a significant difference $[F(2,9)=7 ; p<0.05]$, driven by a significant difference between the fully trained and the classroom only groups $(M=55$ changes vs. $M=83.3$ changes, $p<0.01)$.

The difference in brake applications between the groups six weeks post-course was not significant $[F(2,9)=3.5 ; p>0.05]$, though post-hoc testing revealed a statistically significant difference between the full course group and the control group $(M=18.8$ applications vs. $M=27$ applications; $p<0.05)$.

Again there was very little variation (and no statistical difference) in the average time taken to complete the circuit.

Data was also collected for following distance to the vehicle in front, how often the driver exceeded $1800 \mathrm{rpm}$, and how often the driver did not seem to be looking far enough down the 
stream of traffic to anticipate changes in traffic conditions. There were too few occurrences of any of these events in any of the groups to make for a useful analysis

At the conclusion of the three month assessment period focus groups were conducted with the drivers who had participated in the full training program. While they each had many years of driving experience, they all believed the new driving style was worthwhile and were confident that they had reduced their fuel consumption on a day-to-day basis. The positive outcome was somewhat surprising to some of the drivers, who held a pre-conceived notion that the course would not really deliver much difference in their driving style or fuel consumption. Typical statements included:

Instead of fighting the flow of traffic you are flowing with it Less hectic behind the wheel Seems smoother in the cab I feel like I am keeping the vehicle flowing rather than a lot of stopping and starting

\section{DISCUSSION}

While some differences approached rather than reached statistical significance, the overall pattern of results points to a substantial saving in fuel, gear changes and braking applications for those drivers who completed the full ecodrive training course. These positive outcomes relate to the comparison of results pre- and post-course for the fully trained drivers, as well as the comparison between the fully trained drivers and a control group six weeks after the training. Importantly, the benefits were not lost after 12 weeks, and critically the savings did not necessitate a sacrifice in travelling time or overall travel speed.

It is puzzling why the classroom-only group did not also improve. The only difference between this group and the fully trained drivers was that the latter completed a circuit test drive immediately before and immediately after the training course. Ordinarily the trainer would use these sessions to provide feedback to the drivers from the passenger seat to consolidate the lessons of the course. However, in the trial the accompanying observer provided no feedback. Given their level of experience, however, the drivers likely facilitated their own consolidation. It should be noted that the two groups completed the course separately, though the content, instructor, teaching aids and duration were the same. While the instructor was not blinded to driver group allocation, he had a vested interest in both groups delivering superior performance relative to the control group.

It would seem that either or both of the circuit drives on the day of the course (pre- and postcourse) are important for achieving the fuel consumption improvements, despite the fact that no feedback was provided by the in-cab observers. Many or all of the classroom-only drivers drove a heavy vehicle on the day of the course anyway as part of their job, so just driving on the day of the course would not seem to be the critical element. Perhaps the immediacy and connection of driving the circuit after the course is essential for success. Additional research is needed to establish how and why the pre- and post-training driving seem to be critical to success, particularly since other studies have provided no more than a one-hour video as the training and yet still found a 3\% fuel economy benefit (e.g. Parkes \& Reed, 2005). 
The measures of road safety - following distance and reading the downstream traffic - did not yield useful data in that there were too few instances of "unsafe" driving in all groups. Given the handling characteristics of a fully-laden 68 tonne B-double heavy vehicle this outcome should perhaps not be surprising. It is also possible that the presence of an observing passenger may lead to a moderation of driving behaviour (note that since the observer was present for all drivers across all groups this can not be a confounding variable in the data). More useful safety data could be derived from in-service driving in a longer term study and using data loggers to automatically record the variables of interest.

The magnitude of the reductions in fuel consumption and the retention of the ecodriving skill by the trained drivers over the 12 weeks, and for some variables a progressive improvement, suggests that this form of training could play a valuable role in reducing vehicle fuel consumption and related emissions. The employer who provided the trucks and drivers for the trial uses approximately 1.5 million litres (almost 400,000 gallons) of diesel fuel per annum even a reliable $1 \%$ reduction in consumption would translate into a financial saving of about $\$ 15,000$ and a reduction of about 40 tonnes of $\mathrm{CO}_{2}$, per annum - the results reported here suggest up to $27 \%$ savings are possible. Further, the reductions in gear changes and brake applications and a smoother driving style would be expected to have implications for vehicle repair and maintenance costs as well as for driver fatigue, and in turn these variables would positively impact road safety and occupational health and safety outcomes. Indeed, in post-trial discussions the fully trained drivers noted, unprompted, the reductions in stress and fatigue in their new driving style. Recognising these benefits means that the drivers need not be encouraged to adopt or maintain the new driving style simply to save their employer in fuel costs.

While very promising, the results of the pilot field trial need to be interpreted with some caution. The outcomes are certainly in line with previous European findings (e.g. Holcim, 2005), though they are at the upper end. It is not known whether the results will transfer to non-experimental conditions or whether the transfer will be at the same level. The use of a test circuit and the presence of an observer in the cab recording data are somewhat artificial. However, all of the trial and control drivers used the same circuit, which was on the open road in ordinary traffic conditions, and all were accompanied by an observer, and so neither of these contributed to the differences found between the groups in the trial. The individual variability within driver performance is quite large, and the small group sizes used here, governed primarily by logistical constraints, is not enough to smooth that variability.

A larger trial is certainly warranted, making use of automated in-service monitoring to log fuel use, gear changes, brake applications and so on, in everyday driving. Such devices are already in use in heavy vehicles and are relatively inexpensive, though anecdotally we know that in many fleets the features of these devices are generally under-utilised, particularly when a central monitoring company might charge extra for additional detail or analysis of the data. A larger range of road safety variables could also be assessed with a longitudinal, in-service study. For example, linking accelerometers into a GPS-based monitoring system would allow for heavy acceleration and braking and swerving to be logged and analysed as a function of type of road, time of day and so on. 
In plans for a larger trial we also propose to investigate the usefulness of in-cab driver feedback. A number of commercial options exist to feed back to the driver variables such as fuel consumption, particularly for cars. However, there would seem to be a lack of research into the mode of feedback most suitable and useful to truck drivers, or indeed the amount and type of information that should be made available to the driver, and whether drivers will make use of it even if it is provided.

Instantaneous in-cab driver feedback may prove to be a reinforcer itself for behaviour change for some drivers, but it is likely that a more tangible reward will be required. Competition is a feature of driving for many drivers, whether it is speed, first to arrive, 'making' a set of traffic lights as they cycle to a red phase, etc. The same instinct may be harnessed to encourage efforts to save fuel. A commercial operation based in Germany, FleetBoard (www.fleetboard.com) fits a telematics system to Mercedes heavy vehicles to automatically report in-service variables such as fuel consumption and location for fleet and logistics management functions. An ecodrive-type performance is also available for each driver on the internet to enable individuals to strive to improve their own profile or to make comparisons with the performance of other drivers. The company suggests that it is realistic for a driver to save two litres of fuel over 100 kilometres (or $1 / 2$ a gallon over 62 miles). In 2008 the company's 'Driver's League' involved more than 4,000 drivers, twenty of whom had been elevated to 'Master Class' status to compete for a racing event package. The winning driver's company wins free hire of a Mercedes truck, along with the positive exposure. While a rigorous evaluation does not seem to have been conducted, the arrangement seems popular for both drivers and their employers.

\section{ACKNOWLEDGEMENTS}

We acknowledge the collaboration of the Cement Industry Federation, Blue Circle Southern Cement, and Sustainability Victoria, as well as Australia's Commonwealth Department of Infrastructure, Transport Regional Development \& Local Government for funding this study.

\section{REFERENCES}

ABS (2008). Survey of motor vehicle use, Australia, 12 months ended 31 Oct 2007. Australian Bureau of Statistics report 9208.0 Canberra: Commonwealth of Australia. http://www.abs.gov.au

af Wåhlberg, A.E. (2007b). Fuel efficient driving training - state of the art and quantification of effects. Viewed 15 November 2007. http://www.ecodrive.org/Downloads.203.0.html

Coyle, M., \& Brown, S. (2004). Practical measures to improve fuel efficiency and reduce environmental impact that can easily be applied in road freight transport operation. Presented at the Windsor Workshop, Ontario, 14-17/6/04. Online at http://www.imise.co.uk/assets/WindsorWorkshop.pdf

Holcim. (2005). Diesel club - one possible measure - aim to reduce fuel consumption and CO2 emissions by $5 \%$. Presentation to a Logistics Forum. Antwerp.

Parkes, A.M., \& Reed, N. (2005). Fuel efficiency training in a full-mission truck simulator. In Department for Transport (Ed.), Behavioural Research in Road Safety 2005: Fifteenth Seminar (pp. 135-146). London: Department for Transport. 
SAFED (2007). Save and fuel efficient driving. Accessed online http://www.safed.org.uk on $30 / 7 / 08$.

Zarkadoula, M., Zoidis G. \& Tritopoulou, E. (2007). Training urban bus drivers to promote smart driving: A note on a Greek eco-driving pilot program. Transportation Research Part D: Transport and Environment, 12(6), 449-451. 